Original Research Paper

\title{
Some Generalized Fixed Point Theorems of Contraction Type Mappings in Quasi Metric Spaces
}

\author{
Sahar Mohamed Ali Abou Bakr \\ Department of Mathematics, Faculty of Science, Ain Shams University, Cairo, Egypt
}

Article history

Received: 31-05-2017

Revised: 29-08-2017

Accepted: 6-10-2017

Email: saharm ali@yahoo.com; saharm_ali@sci.asu.edu.eg

\begin{abstract}
This paper adjusts conditions on new defined given the name $\{a, d, c ; r\}$ ctype of contraction mappings on complete quasi metric space, confirms that there is only one fixed of any of these mappings with these adjustments, extends and generalizes results given in some previous research papers, and then builds a convergence theorem for a sequence of fixed points of $\left\{S_{n}\right\}_{n \in N} ;\left\{w_{n}^{*}\right\}_{n \in N}$ to the unique fixed of $S ; w$, provided that $\lim _{n \rightarrow \infty} S_{n}(w)=S(w)$.
\end{abstract}

Keywords: Fixed Point Theorem, Generalized $\{a, b, c\}$ - Contraction and Nonexpansive Mappings

\section{Introduction}

\section{Preliminaries}

Generalizations of Banach contraction principle (Banach, 1922) have been established in various settings. Alber and Guerre-Delabriere (1997) introduced the definition of weakly contractive mappings and they proved some fixed point theorems in case of Hilbert spaces, Rhoades in (Rhoades, 1977; 2001) give extended results of (Alber and Guerre-Delabriere, 1997) to complete metric spaces, and more discussed concepts shown in (Hardy and Rogers, 1973); (Sehie, 1980), and (Sahar Mohamed Ali Abou Bakr, 2013).

In this paper $\mathrm{X}$ will be a nonempty set and $S$ be mapping from $X$ into $X$, we have:

\section{Definition 1}

If $X$ is normed space, we have:

(1) If there are reals $a, b, c \in[0,1]$ with $a+b+c<1$ such that:

$$
\begin{gathered}
\|S(u)-S(v)\| \leq a\|u-v\|+b\|v-S(v)\| \\
+c\|u-S(u)\| \forall u, v \in X
\end{gathered}
$$

then $\mathrm{S}$ is $\{a, b, c\}$ generalized contraction on $X$.

(2) If there are reals $a, b, c \in[0,1]$ with $a+b+c=1$ such that:

$$
\begin{gathered}
\|S(u)-S(v)\| \leq a\|u-v\|+b\|v-S(v)\| \\
+c\|u-S(u)\| \forall u, v \in X
\end{gathered}
$$

then $S$ is $\{a, b, c\}$ generalized nonexpansive.
(3) If there are reals $a, b, c \in[0,1]$ with $0 \leq c<\frac{1}{2}$ and $a$ $+b+c<1$ such that:

$$
\begin{array}{r}
\|S(u)-S(v)\| \leq a\|u-v\|+b\|v-S(v)\| \\
+c \max \{\|u-S(u)\|,\|x-S(y)\|\}
\end{array}
$$

for all $u, v \in X$, then $S$ is $\{a, b, c\}$-ctype mapping.

(4) If there are reals $a, b, c \in[0,1]$ with

$0<a<1,0<b, 0 \leq c<\frac{1}{2}$ and $a+b+c=1$ such that:

$$
\begin{array}{r}
\|S(u)-S(v)\| \leq a\|u-v\|+b\|v-S(v)\| \\
+c \max \{\|u-S(u)\|,\|u-S(v)\|\}
\end{array}
$$

for all $u, v \in X$, then $S$ is $\{a, b, c\}$-ntype mapping.

El-Shobaky et al. (2007) proved that there is only one fixed point $(f p)$ of $\{a, b, c\}$-generalized contraction mapping defined on closed and convex subset of weakly Cauchy normed space $X$. The existence of only one $f p$ of $\{a, b, c\}$-generalized non expansive mapping when $C$ contains contraction point proved in (Sahar Mohamed Ali Abou Bakr, 2009). Fixed point theorems established for $\{a, b, c\}$-ctype and ntype in (Sahar Mohamed Ali Abou Bakr, 2013). Mainly; we are interested in:

\section{Theorem 1}

Sahar Mohamed Ali Abou Bakr (2013) Let $X$ be a Banach space $X, S$ be $\{a, b, c\}$-ctype mapping, then $S$ has only one $f p$. Moreover, for any $u \in X$, the iterated sequence $\left\{S^{n}(u)\right\}_{n \in N}$ is convergent to the $f p$ of $S$. 
In case of quasi-metric spaces, we have:

Definition 2 Sahar Mohamed Ali Abou Bakr (2013)

(1) Let $r$ be a number; $r \geq 1$ and $q$ be a mapping $q: X \rightarrow$ $R+$. Then $(X, q)$ is quasi-metric space iff the following:
(a) $q(u, v)=0$ iff $u=v$.
(b) $q(u, v)=q(v, u)$ for all $u, v \in X$.
(c) $q(u, v) \leq r[q(u, w)+q(w, v)]$ for all $u, v, w \in X$

(2) Let $(X, q)$ be a quasi-metric space and $\left\{v_{n}\right\}_{n \in N}$ be a sequence in $X$. Then $\left\{v_{n}\right\}_{n \in N}$ is

(a) Cauchy iff for every $\epsilon>0$ there is $n_{0}(\epsilon) \in N$ such that:

$$
q\left(v_{n}, v_{m}\right)<\epsilon \forall_{n, m} \geq n_{0}(\epsilon)
$$

(b) Convergent to $v$ iff for every $\epsilon>0$ there is $n_{0}(\epsilon) \in N$ such that:

$$
q\left(v_{n}, v\right)<\epsilon \forall_{n} \geq n_{0}(\epsilon) .
$$

\section{Remark}

It is clear that every metric space is quasi-metric space $(X, q)$ with $r=1$. There are many examples in the literatures of quasi metric spaces which are not metric.

Now, for the purpose of the first result of this paper we define the contraction type of mappings, namely $\{a$, $b, c ; r\}$-contraction type.

\section{Definition 3}

Let $(X, q)$ be a quasi-metric space. Then $S$ that satisfy $q(S(u), S(v)) \leq a q(u, v)+d q(v, S(v))+c \max \{q(u, S(u))$, $q(u, S(v))\}$ for all $u, v \in X$ and some real numbers $a, d$, $c \in[0,1]$ where $a+c<1$ and $r(a+d)+c<1$ is called $\{a, d$, $c ; r$-ctype mapping.

\section{Remark}

Noticed the following:

(1) If $(X, q)$ is a quasi-metric space, $S$ satisfies $q(S(u)$, $S(v)) \leq a q(u, v)+d q(v, S(v))+c q(u, S(u))+e q(u, S(v))$ for all $u, v \in X$ and for some $a, d, c$ and $e \in[0,1], 0 \leq a$ $+(c+e)<1$, and $r(a+d)+c+e<1$, then $S$ is $\{a$, $d, c+e ; r\} g$-ctypel

(2) If $r=1$ the definitions will be reduced to the definition of $\{a, d, c\}$ ctype mappings (Ali, 2013).

(3) The class of $\{a, d, c ; r\}$-ctype mappings is wider than the class of contraction mappings.

\section{Main Results}

We start with some basic lemmas:

\section{Lemma 1} that:

Let $(X, q)$ be quasi-metric and $\left\{v_{n}\right\}_{n \in N}$ be in $X$ such

$q\left(v_{n+2}, v_{n+1}\right) \leq r_{0} q\left(v_{n+1}, v_{n}\right), n=0,1,2, \ldots$

for some positive real number $r_{0}$ with $\mathrm{r} r_{0}<1$. Then $\left\{v_{n}\right\}_{n \in N}$ is Cauchy.

\section{Proof}

We have the following:

$$
\begin{aligned}
q\left(v_{n+2}, v_{n+1}\right) & \leq r_{0} q\left(v_{n+1}, v_{n}\right) \\
& \leq r_{0} r_{0} q\left(v_{n}, v_{n-1}\right)=r_{0}^{2} q\left(v_{n}, v_{n-1}\right)
\end{aligned}
$$

continuing in this process gives:

$q\left(v_{n+2}, v_{n+1}\right) \leq r_{0}^{n+1} q\left(v_{1}, v_{0}\right)$

Now; let $n, m \in N$ be such that $m>n$. We have:

$$
\begin{aligned}
q\left(v_{n}, v_{m}\right) \leq & r\left[q\left(v_{n}, v_{n+1}\right)+q\left(v_{n+1}, v_{m}\right)\right] \\
\leq & r\left[q\left(v_{n}, v_{n+1}\right)+r\left\{q\left(v_{n+1}, v_{n+2}\right)+q\left(v_{n+2}, v_{m}\right)\right\}\right] \\
\leq & r q\left(v_{n}, v_{n+1}\right) r^{2} q\left(v_{n+1}, v_{n+2}\right)+ \\
& +r^{2}\left[r\left\{q\left(v_{n+2}, v_{n+3}\right)\right\}+q\left(v_{n+3}, v_{m}\right)\right] \\
& \leq r q\left(v_{n}, v_{n+1}\right) r^{2} q\left(v_{n+1}, v_{n+2}\right)+r^{3} q\left(v_{n+2}, v_{n+3}\right)+ \\
& +\ldots+r^{m-n} q\left(v_{m-1}, v_{m}\right)
\end{aligned}
$$

Back to inequalities (2.2), we get:

$$
\begin{aligned}
q\left(v_{n}, v_{m}\right) & \leq r r_{0}^{n} q\left(v_{1}, v_{0}\right)+r^{2} r_{0}^{n+1} q\left(v_{1}, v_{0}\right) \\
+ & r^{3} r_{0}^{n+2} q\left(v_{1}, v_{0}\right)+\ldots \\
& +r^{m-n} r_{0}^{n+(m-1-n)} q\left(v_{1}, v_{0}\right) \\
& =r r_{0}^{n}\left[\begin{array}{l}
q\left(v_{1}, v_{0}\right)+r r_{0} q\left(v_{1}, v_{0}\right) \\
+\left(r r_{0}\right)^{2} q\left(v_{1}, v_{0}\right)+\ldots \\
+\left(r r_{0}\right)^{(m-1-n)} q\left(v_{1}, v_{0}\right)
\end{array}\right] \\
& =r r_{0}^{n} q\left(v_{1}, v_{0}\right)\left[\frac{1-\left(r r_{0}\right)^{m-1-n}}{1-r r_{0}}\right] \\
& \leq\left[\frac{r r_{0}^{n}}{1-r r_{0}}\right] q\left(v_{1}, v_{0}\right)
\end{aligned}
$$

Clearly, $r_{0}<1$, taking the limit as $n \rightarrow \infty$ completes the proof. 


\section{Lemma 2}

Let $(X, q)$ be quasi-metric space and $S$ satisfy $q(S(u)$, $S(v)) \leq a q(u, v)+d q(v, S(v))+\mathrm{c} \max \{q(u, S(u), q(u$, $S(v))\}$ for all $u, v \in X$ and for some $a, d, c \in[0,1]$ with $c \neq 1$. Then for any $u \in X,\left\{S^{n}(u)\right\} \mathrm{n} \in \mathrm{N}$ satisfies:

$$
\begin{aligned}
& q\left(S^{n+1}(u), S^{n}(u)\right) \leq\left(\frac{a+d}{1-c}\right) q\left(S^{n}(u), S^{n-1}(u)\right) \\
& q\left(S^{n+1}(u), S^{n}(u)\right) \leq\left(\frac{a+d}{1-c}\right)^{n} q(S(u), u)
\end{aligned}
$$

\section{Proof}

Let $u \in X$, we have:

$$
\begin{aligned}
& q\left(S^{n+1}(u), S^{n}(u)\right) \\
& =q\left(S\left(S^{n}(u)\right), S\left(S^{n-1}(u)\right)\right. \\
& \leq a q\left(S^{n}(u), S^{n-1}(u)\right)+d q\left(S^{n-1}(u), S\left(S^{n-1}(u)\right)\right)+ \\
& +c \max \left\{q\left(S^{n}(u), S\left(S^{n}(u)\right)\right), q\left(S^{n}(u), S\left(S^{n-1}(u)\right)\right)\right\} \\
& \leq(a+d) q\left(S^{n}(u), S^{n-1}(u)\right)+c q\left(S^{n+1}(u), S^{n}(u)\right)
\end{aligned}
$$

This shows that:

$$
(1-c) q\left(S^{n+1}(u), S^{n}(u)\right) \leq(a+d) q\left(S^{n}(u), S^{n-1}(u)\right)
$$

And hence (2.3) is proved. Continuing this inductive process proves (2.4).

We also have:

\section{Theorem 2}

Let $(X, q)$ be a Complete quasi-metric space, $S$ be $\{a$, $d, c, r\}$-ctype mapping. Then $S$ has only one $f p ; y$. Moreover, for any $u \in X,\left\{S^{n}(u)\right\}_{n \in N}$ is converging to $y$.

\section{Proof}

Let $u \in X$ be an arbitrarily chosen element, using Lemma (2) insures that:

$$
\begin{aligned}
& q\left(S^{n+1}(u), S^{n}(u)\right) \leq\left(\frac{a+d}{1-c}\right) \\
& q\left(S^{n}(u), S^{n-1}(u)\right), n=0,1,2, \ldots
\end{aligned}
$$

Since $S$ is $\{a, d, c, r\}$-ctype, we have $r(a+d)+c<1$, consequently $r\left[\frac{a+d}{1-c}\right]<1$. Taking $r_{0}=\left[\frac{a+d}{1-c}\right]$ insures that the inequalities (2.3) of Lemma (1) are satisfied, then we have:

$$
\left.\lim _{n \rightarrow \infty} q\left(S^{n+1}(u), S^{n}(u)\right)\right)=0
$$

and $\left\{S^{n}(u)\right\}_{n \in N}$ is Cauchy. Since $X$ is complete, $\left\{S^{n}(u)\right\}_{n \in N}$ is converging to some element $y \in X$ :

$\lim _{n \rightarrow \infty} q\left(S^{n}(u), y\right)=0$

On the other side:

$$
\begin{aligned}
& q\left(S(y), S^{n+1}(u)\right) \geq a q\left(y, S^{n}(u)\right) \\
& +d q\left(S^{n}(u), S n+1(u)\right) \\
& +c \max \left\{q(y, S(y)), q\left(y, S^{n+1}(u)\right)\right\}
\end{aligned}
$$

Accordingly, we have:

$$
\begin{aligned}
& q(S(y), y) \leq r q\left(S(y), S^{n+1}(u)\right)+r q\left(S^{n+1}(u), y\right) \\
& \leq r\left[\begin{array}{l}
a q\left(y, S^{n}(u)\right)+d q\left(S^{n}(u), S^{n+1}(u)\right) \\
+c \max \left\{q(y, S(y)), q\left(y, S^{n+1}(u)\right)\right\}
\end{array}\right]+r q\left(S^{n+1}(u), y\right)
\end{aligned}
$$

Taking the limit as $n \rightarrow \infty$ with the use of (2.5) and (2.6) prove:

$$
q(S(y), y) \leq c q(y, S(y))
$$

If $q(S(y), y) \neq 0$, then $1 \leq \mathrm{c}$, this yields $q(S(y), y)=0$, consequently $S(y)=y$.

Finally to prove that there is only one of such a point, let $y$ and $z$ be two distinct points of $S$ such that $S(y)=y$ and $S(z)=z$. Then the following strict inequality gives an obvious contradiction:

$$
\begin{aligned}
q(y, z) & =q(S(y), S(z)) \\
& \leq a q(y, z)+d q(z, S(z)) \\
& +c \max \{q(y, S(y)), q(y, S(z))\} \\
= & (a+c) q(y ; z) \\
< & q(y ; z) .
\end{aligned}
$$

\section{Corollary 1}

Let $(X, q)$ be complete and $S$ satisfy:

$$
\begin{aligned}
q(S(u), S(v)) & \leq a q(u, v)+d q(v, S(v)) \\
& +c q(u, S(u))+e q(u, S(v))
\end{aligned}
$$

for all $u, v \in X$ and for some $a, d, c$ and $e \in[0,1], 0 \leq a+c$ $+e<1$, and $r(a+d)+c+e<1$. Then $S$ has only one $f p$.

\section{Proof}

Let $S$ satisfy $q(S(u), S(v)) \leq a q(u, v)+d q(v, S(v))+c q(u$, $S(u))+e q(u, S(v))$ for all $u, v \in X$ and for some real numbers $a, d, c$ and $e \in[0,1], r(a+d)+c+e<1$. Then 
$q(S(u), S(v)) \leq a q(u, v)+d q(v, S(v))+(c+e) \max \{q(u$, $S(u)), q(u, S(v))\}$ for all $u, v \in C$, since $r(a+d)+(c+e)$ $<1, S$ is $\{a, d, c+e\}$-ctype mapping, using Theorem (1) proves that $\mathrm{S}$ has only one $f p$.

In some situations it is difficult to determine the fixed point of $\{a, d, c, r\}$ types of mapping, approximating such a fixed point is va-porable tool. For this direction we have the following result.

\section{Theorem 3}

If $\left\{S_{n}\right\}_{n \in N}$ and $S$ are $\{a, d, c, r\}$-ctype of mappings on the complete quasi metric space $(X, q), w \mathrm{n}$ is the unique fixed of each $S_{n}, S_{n}\left(w_{n}^{*}\right)=w_{n}^{*}$ and $\left\{S_{n}\left(w_{n}^{*}\right)\right\}_{n \in N}$ converges to $w^{*}$,

$$
\lim _{\mathrm{n} \rightarrow \infty} \mathrm{q}\left(\mathrm{S}_{\mathrm{n}}\left(\mathrm{w}^{*}\right), \mathrm{S}\left(\mathrm{w}^{*}\right)\right)=0,
$$

where, $w^{*}$ is the unique fixed of $S ; S\left(w^{*}\right)=w^{*}$, then $\left\{w_{n}^{*}\right\}_{n \in N}$ converges to $w^{*}$ :

$$
\lim _{n \rightarrow \infty} q\left(w_{n}^{*}, w_{n}^{*}\right)=0
$$

\section{Proof}

We have:

$$
\begin{aligned}
q\left(w_{n}^{*}, w^{*}\right) & =q\left(S_{n}\left(w_{n}^{*}\right), S\left(w^{*}\right)\right) \\
& \leq r\left[q\left(S_{n}\left(w_{n}^{*}\right), S\left(w_{n}^{*}\right)\right)+q\left(S\left(w_{n}^{*}\right), S\left(w^{*}\right)\right)\right]
\end{aligned}
$$

Because of contraction condition we have:

$$
\begin{aligned}
q\left(S\left(w_{n}^{*}\right), S\left(w^{*}\right)\right) \\
\quad \leq a q\left(w_{n}^{*}, w^{*}\right)+d q\left(w_{n}^{*}, S\left(w_{n}^{*}\right)\right)+ \\
\quad+c \max \left\{q\left(w^{*}, S\left(w^{*}\right)\right), q\left(w^{*}, S\left(w_{n}^{*}\right)\right)\right\} \\
\quad \leq a q\left(w_{n}^{*}, w^{*}\right)+d q\left(S_{n}\left(w_{n}^{*}\right), S\left(w_{n}^{*}\right)\right)+ \\
\quad+c \max \left\{q\left(w^{*}, w^{*}\right), q\left(w^{*}, S\left(w_{n}^{*}\right)\right)\right. \\
\quad \leq a q\left(w_{n}^{*}, w^{*}\right)+d q\left(S_{n}\left(w_{n}^{*}\right), S\left(w_{n}^{*}\right)\right)+ \\
\quad+c q\left(S\left(w^{*}\right), S\left(w_{n}^{*}\right)\right)
\end{aligned}
$$

Inequalities (2.8) shows that:

$$
q\left(S\left(w_{n}^{*}\right), S\left(w^{*}\right)\right) \leq \frac{1}{1-c}\left[a q\left(w_{n}^{*}, w^{*}\right)+d q\left(S_{n}\left(w_{n}^{*}\right), S\left(\left(w_{n}^{*}\right)\right)\right]\right.
$$

Using (2.9) in (2.7) gives:

$$
\begin{aligned}
q\left(w_{n}^{*}, w^{*}\right) & \leq r q\left(S_{n}\left(w_{n}^{*}\right), S\left(w_{n}^{*}\right)\right)+ \\
& +\frac{r}{1-c}\left[\begin{array}{l}
a q\left(w_{n}^{*}, w^{*}\right) \\
+d q\left(S_{n}\left(w_{n}^{*}\right), S\left(w_{n}^{*}\right)\right)
\end{array}\right]
\end{aligned}
$$

Inequalities (2.10) shows that:

$$
\begin{aligned}
& q\left(w_{n}^{*}, w^{*}\right) \leq\left|\frac{1-c}{1-c-r a}\right| r\left[1+\frac{d}{1-c}\right] \\
& q,\left(S_{n}\left(w_{n}^{*}\right), S\left(w_{n}^{*}\right)\right)
\end{aligned}
$$

Hence:

$$
q\left(w_{n}^{*}, w^{*}\right) \leq\left|\frac{(1-c+d) r}{1-c-r a}\right| q,\left(S_{n}\left(w_{n}^{*}\right), S\left(w_{n}^{*}\right)\right)
$$

Since:

$$
\begin{aligned}
& q\left(S_{n}\left(w_{n}{ }^{*}\right), S\left(w^{*}{ }_{n}\right)\right) \\
& \leq r\left[q\left(S_{n}\left(w_{n}{ }^{*}\right), S_{n}\left(w^{*}\right)\right)+q\left(S_{n}\left(w^{*}\right), S\left(w_{n}^{*}\right)\right)\right] \\
& \leq r q\left(S_{n}\left(w_{n}{ }^{*}\right), S_{n}\left(w^{*}\right)\right)+ \\
& +r^{2} q\left(S_{n}\left(w^{*}\right), S\left(w^{*}\right)\right)+r^{2} q\left(S\left(w^{*}\right), S\left(w^{*}{ }_{n}\right)\right)
\end{aligned}
$$

Using (2.9) in (2.12):

$$
\begin{aligned}
& q\left(S_{n}\left(w_{n}^{*}\right), S\left(w_{n}^{*}\right)\right) \\
& \leq r q\left(S_{n}\left(w_{n}^{*}\right), S_{n}\left(w^{*}\right)+r^{2} q\left(S_{n}\left(w^{*}\right), S\left(w^{*}\right)\right)\right. \\
& +\frac{r^{2}}{1-c}\left[a q\left(w_{n}^{*}, w^{*}\right)+d q\left(S_{n}\left(w_{n}^{*}\right), S\left(w_{n}^{*}\right)\right)\right]
\end{aligned}
$$

Inequalities (2.13) proves:

$$
\begin{aligned}
& q\left(S_{n}\left(w_{n}^{*}\right), S\left(w_{n}^{*}\right)\right) \leq\left[\frac{1-c}{1-c-r^{2} d}\right] \\
& {\left[\begin{array}{l}
r q\left(S_{n}\left(w_{n}^{*}\right), S_{n}\left(w^{*}\right)\right)+ \\
+r^{2} q\left(S_{n}\left(w^{*}\right), S\left(w^{*}\right)\right)+\frac{a r^{2}}{1-c} q\left(w_{n}^{*}, w^{*}\right)
\end{array}\right]}
\end{aligned}
$$

On the other hand:

$$
\begin{aligned}
q\left(S_{n}\left(w_{n}^{*}\right), S_{n}\left(w^{*}\right)\right) \\
\quad \leq a q\left(w_{n}^{*}, w^{*}\right)+d q\left(w_{n}^{*}, S_{n}\left(w^{*}\right)\right)+ \\
\quad+c \max \left\{q\left(w_{n}^{*}, S_{n}\left(w_{n}^{*}\right)\right), q\left(w_{n}^{*}, S_{n}\left(w^{*}\right)\right)\right\} \\
\quad \leq a q\left(w_{n}^{*}, w^{*}\right)+d q\left(S\left(w^{*}\right), S_{n}\left(w^{*}\right)\right)+ \\
\quad+c \max \left\{q\left(w_{n}^{*}, w_{n}^{*}\right), q\left(S_{n}\left(w_{n}^{*}\right), S_{n}\left(w^{*}\right)\right)\right\} \\
\quad \leq a q\left(w_{n}^{*}, w^{*}\right)+d q\left(S\left(w^{*}\right), S_{n}\left(w^{*}\right)\right)+ \\
\quad+c q\left(S_{n}\left(w_{n}^{*}\right), S_{n}\left(w^{*}\right)\right) \\
\quad+c q\left(S\left(w^{*}\right), S\left(w^{*}\right)\right)
\end{aligned}
$$

Consequently: 


$$
\begin{aligned}
& q\left(S_{n}\left(w_{n}^{*}\right), S_{n}\left(w^{*}\right)\right) \leq\left[\frac{1}{1-c}\right] \\
& \quad\left[a q\left(w_{n}^{*}, w^{*}\right)+d q\left(S\left(w^{*}\right), S_{n}\left(w^{*}\right)\right)\right]
\end{aligned}
$$

Using (2.16) in (2.14):

$$
\begin{aligned}
& q\left(S_{n}\left(w_{n}^{*}\right), S\left(w_{n}^{*}\right)\right) \leq\left[\frac{1-c}{1-c-r^{2} d}\right] \\
& \left\{\begin{array}{c}
\left(\frac{r}{1-c}\right)\left[a q\left(w_{n}^{*}, w^{*}\right)+d q\left(S\left(w^{*}\right), S_{n}\left(w^{*}\right)\right)\right] \\
+r^{2} q\left(S_{n}\left(w^{*}\right), S\left(w^{*}\right)\right)+\left(\frac{a r^{2}}{1-c}\right) q\left(w_{n}^{*}, w^{*}\right)
\end{array}\right\} \\
& q\left(S_{n}\left(w_{n}^{*}\right), S\left(w_{n}^{*}\right)\right) \leq\left[\frac{r a(1+a)}{\left(1-c-r^{2} d\right)}\right] q\left(w_{n}^{*}, w^{*}\right)+ \\
& +\left[\frac{1-c}{1-c-r^{2} d}\right]\left[\left(\frac{r d}{1-c}\right)+r^{2}\right] q\left(S\left(w^{*}\right), S_{n}\left(w^{*}\right)\right)
\end{aligned}
$$

Using (2.18) in (2.11) yields:

$$
\begin{aligned}
& q\left(w_{n}^{*}, w_{n}^{*}\right) \leq\left[\frac{(1-c+d) r}{1-c-r a}\right]\left\{\left[\frac{r a(1+a)}{\left(1-c-r^{2} d\right)}\right] q\left(w_{n}^{*}, w^{*}\right)+\right. \\
& \left.+\left[\frac{1-c}{1-c-r^{2} d}\right]\left[\left(\frac{r d}{1-c}\right)+r^{2}\right] q\left(S\left(w^{*}\right), S_{n}\left(w^{*}\right)\right)\right\} \\
& \leq\left[\frac{(1-c+d) r}{1-c-r a}\right]\left[\frac{r a(1+a)}{\left(1-c-r^{2} d\right)}\right] q\left(w_{n}^{*}, w_{n}^{*}\right)+ \\
& +\left[\frac{(1-c+d) r}{1-c-r a}\right]\left[\frac{1-c}{1-c-r^{2} d}\right]\left[\left(\frac{r d}{1-c}\right)+r^{2}\right] q\left(S\left(w^{*}\right), S_{n}\left(w^{*}\right)\right)
\end{aligned}
$$

Consequently:

$$
\begin{gathered}
q\left(w_{n}^{*}, w^{*}\right) \leq\left[\frac{1}{1-\left[\frac{(1-c+d) r}{1-c-r a}\right]\left[\frac{r a(1+a)}{1-c-r^{2} d}\right]}\right. \\
{\left[\frac{(1-c+d) r}{1-c-r a}\right]} \\
{\left[\frac{1-c}{1-c-r^{2} d}\right]\left[\left(\frac{r d}{1-c}\right)+r^{2}\right] q\left(S\left(w^{*}\right), S_{n}\left(w^{*}\right)\right)}
\end{gathered}
$$

Taking the limit as $n \rightarrow \infty$ yields that $\lim _{n \rightarrow \infty} q\left(w_{n}^{*}, w^{*}\right)=0$ because of the given assumption $\lim _{n \rightarrow \infty} q\left(S_{n}\left(w^{*}\right), S\left(w^{*}\right)\right)=0$. This completes the proof.

\section{Conclusion}

This paper adjusts conditions on new defined contraction type of mapping; namely $\{a, d, c, r\}$-ctype on quasi metric space, checked the validation of existence of unique fixed point of such type, gives a generalization of theorem (1) with these adjustments, shows that Corollary (1) extends some of the results given in (El-Shobaky et al., 2007; Gregus, 1980; Wong, 1975) and then builds a convergence theorem for sequence of fixed points of $\left\{S_{n}\right\}_{n \in N} ;\left\{w_{n}^{*}\right\}_{n \in N}$ to the unique fixed point of $S ; w$ under the assumption $\lim _{n \rightarrow \infty} S_{n}(w)=w$.

\section{Acknowledgment}

Sincer thanks to the reviwer valable comments.

\section{Conflict of Interests}

The author has no interesting conflict of interest.

\section{References}

Alber, Y.I. and S. Guerre-Delabriere, 1997. Principles of Weakly Contractive Maps in Hilbert Spaces. New Resulty in Operator Theory, Gohberg. I. and Y, Lyubich (Eds)., pp: 7-22.

Banach, S., 1922. Sur les operations dans les ensembles absrtacts et leurs applications. Fun. Math., 3: 133-81.

Czerwik, S., 1993. Contraction Mapping in b-Metric Spaces. Acta Mathematics et Information Universitatis Ostraviensis 1: 5-11.

El-Shobaky, E.M., S. Mohamed Ali and M.S. Ali, 2007. Generalization of Banach contraction principle in two directions. Int. J. Math. Statistics, 3: 112-115. DOI: $10.3844 /$ jmssp.2007.112.115

Gregus, M., 1980. A fixed point theorem in bannach spaces. Bollettion, Unione mathematica Italiana A. Serie V 17: 193-198.

Hardy, G.E. and T.D. Rogers, 1973. A generalization of a fixed point theorem of Reich. Canada. Math. Bul., 16: 201-206. DOI: 10.4153/CMB-1973-036-0

Rhoades, B.E., 1977. A comparison of variuos definitions of contractive map-pings. Trans. Amer. Math. Soc., 226: 257-290.

DOI: 10.1090/S0002-9947-1977-0433430-4

Rhoades, B.E., 2001. Some theorems on weakly contractive maps. Nonlinear Anal., 47: 2683-2693. DOI: 10.1016/S0362-546X(01)00388-1

Sahar Mohamed Ali Abou Bakr, S.M., 2013. Functional Analysis with Some Fixed Points Theories' Approaches. Verlag/publisher Lab Lambert Academic Publishing, Deutschland/ Germany.

Sahar Mohamed Ali Abou Bakr., 2009. On Fixed Point Theorem of $\{a, b, c\}$-Generalized Nonex-pansive Mappings in Normed Spaces. J. Egyptian Math. Society, 17: 1-13.

Sahar Mohamed Ali Abou Bakr., 2013. Fixed point theorem of $\{a, b, c\}$ contraction and non-expansive type mappings in weakly Cauchy normed spaces. Anal. Theory Appl., 29: 280-288. 
Sahar Mohamed Ali Abou Bakr., 2013. Fixed point theorems of some type contraction map-pings. J. Nonlinear Convex Analysis Tokyo Japan, 14: 331-342.

Sehie, P., 1980. On general contraction-type conditions. J. Korean Math. Soc., 17: 131-140.
Wong, C.S., 1975. On Kannan maps. Proceeding Am. Math. Society, 47: 105-116.

DOI: $10.1090 / \mathrm{S} 0002-9939-1975-0358468-0$ 